\title{
Mécanisme multiphysique de dégradation des zircones orthopédiques : couplage entre vieillissement hydrothermal et frottement en simulateur de hanche
}

\section{Multi-physics degradation mechanisms of orthoaedic zirconia: Coupling hydrothermal ageing and wear on a hip simulator} \author{
et Julia Espinouse ${ }^{3}$ \\ ${ }^{1}$ MATEIS, INSA-Lyon, 20 avenue Einstein, 69621 Villeurbanne, France \\ 2 SERF, 85 avenue des Bruyères, 69153 Décines, France \\ ${ }^{3}$ Medical Group, 5 chemin du Catupolan, 69120 Vaulx en Velin, France
}

Laurent Gremillard ${ }^{1}$, Laura Martin ${ }^{1}$, Jérôme Chevalier $^{1}$, Jean-Luc Aurelle ${ }^{2}$

\begin{abstract}
The degradation of zirconia-based ceramic components for total hip arthroplasty (head and cup) has been the topic of many works. However, the correlation between what is measured in vivo and what is expected from in vitro simulations on hip simulators may be sometimes feeble, especially where zirconia component are concerned, mainly due to a lack of representativeness of in-vitro experiments. We seek here to explore the combined effects of hydrothermal ageing and wear on zirconia components. We will show that hydrothermal ageing increases the roughness of zirconia components, which in turns might increase the wear rate of the polyethylene counterparts. Moreover, the friction during hip simulation increases the ageing rate of the zirconia components. This auto-accelerating degradation may explain some of the poor long-term in-vivo results of zirconia hip prostheses reported in the literature. Finally, we will show that zirconia-toughened alumina components may be free from this combined degradation.
\end{abstract}

Résumé. Malgré les nombreuses recherches menées sur la dégradation des composants de prothèses de hanches à base de zircone (tête et cupule), on constate toujours une corrélation assez faible entre les observations in vivo et les simulations in vitro (sur simulateurs de marche par exemple), principalement due à un manque de représentativité des conditions de test. Lors de ce travail, nous examinons les effets combinés du vieillissement hydrothermal et du frottement sur les composants en zircone et composites alumine-zircone. Nous montrons que le vieillissement hydrothermal augmente la rugosité des têtes en zircone, ce qui pourrait augmenter le taux d'usure des cupules en polyéthylène frottant contre ces têtes. De plus, le frottement occasionné par les tests en simulateur de marche accélère le vieillissement des têtes en zircone. Ces mécanismes de dégradation auto-accélérés pourraient expliquer quelques-uns des mauvais résultats in vivo à long terme des prothèses de hanche en zircone. Pour finir, nous montrons que les composites alumine-zircone peuvent ne pas être affectés par ce mécanisme.

\section{INTRODUCTION}

Plus de 4 millions d'implants céramiques ont été implantés depuis l'introduction de l'alumine en orthopédie dans les années 1960. Le succès clinique est au rendez-vous, puisque les prothèses de hanches dans lesquelles une tête en céramique frotte contre une cupule en polyéthylène offrent les plus bas taux de révisions comparés aux systèmes métal-métal et métal-polyéthylène. Le problème principal des premiers implants céramiques était leur fragilité intrinsèque, qui limite l'usage des céramiques aux dimensions d'implants pour lesquelles les contraintes mécaniques en fonctionnement sont moindres. C'est encore le cas pour l'alumine, bien moins pour les nouveaux composites alumine-zircone type Biolox Delta. Moyennant quoi, le taux de rupture des implants céramique est aujourd'hui inférieur à $0,01 \%$.

L'enjeu majeur des prothèses orthopédique est maintenant l'augmentation de leur durée de vie, en raison à la fois de l'augmentation de l'espérance de vie des patients et de la pose chez des patients de plus en plus jeunes. Ce n'est plus un problème purement «matériaux», puisque la cause principale de révision d'une prothèse de hanche est le descellement aseptique (responsable de $58 \%$ des échecs d'après les registres anglais et suédois [1]). Le descellement aseptique est dû le plus souvent au relargage de débris d'usure au voisinage de la prothèse, qui entraînent une inflammation résultant en une ostéolyse.

La zircone est un cas «d'école». Cette céramique offre les meilleures propriétés mécaniques parmi les oxydes céramiques monolithiques, grâce à son mécanisme de renforcement par transformation de phase (la phase quadratique (métastable) retenue après frittage se transforme en phase monoclinique en présence d'une contrainte, par exemple en front de fissure, et l'augmentation de volume qui en résulte superpose des contraintes de compression au champ de contrainte existant, ralentissant la propagation de la fissure et donc renforçant le matériau. Le mauvais côté de la métastabilité de la phase quadratique est que le changement de phase peut aussi se produire en surface en présence d'eau. Ceci entraîne alors une augmentation de 


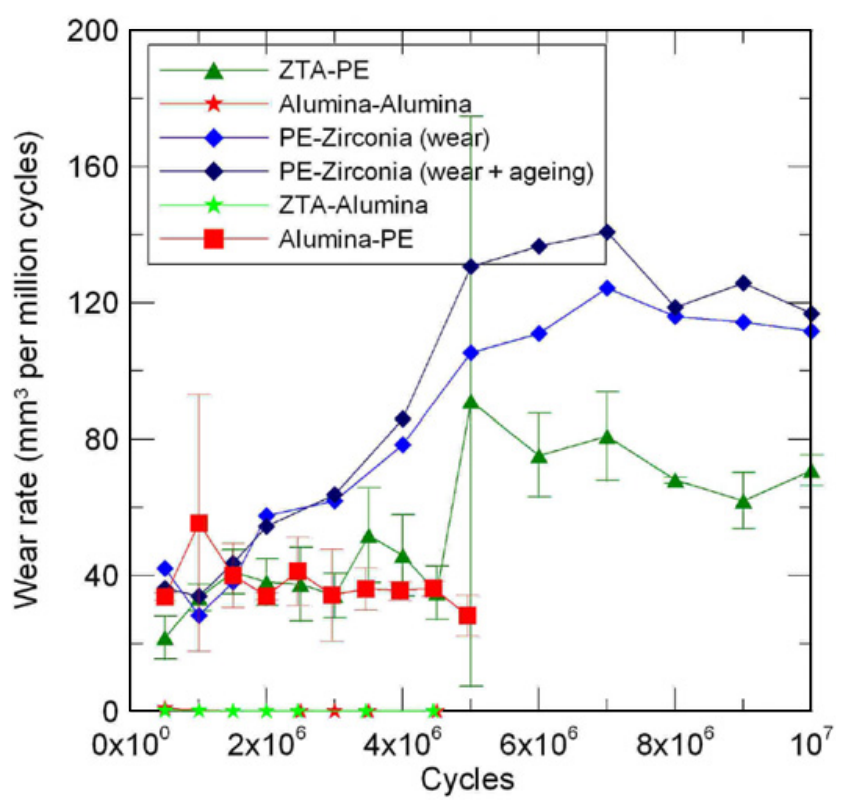

Figure 1. Taux d'usure des différents couples de frottement.

la rugosité et l'apparition de microfissuration permettant à l'eau d'accéder au volume du matériau et à la transformation de se propager vers des couches de plus en plus profondes. Ce phénomène est appelé «vieillissement hydrothermal»(ageing ou Low Temperature Degradation). Le vieillissement hydrothermal augmente graduellement avec le temps, pour des durées comparables avec les durées d'implantation.

Le but de ce travail est d'examiner les effets croisés $\mathrm{du}$ vieillissement hydrothermal et du frottement sur la dégradation des têtes en zircone.

\section{PROCÉDURES EXPÉRIMENTALES}

Les effets du vieillissement et du frottement sont testés sur 3 têtes en zircone 3Y-TZP (têtes Z, fournies par SERF) et 3 têtes prototypes en composite alumine-zircone (têtes ZTA, fournies par Medical Group), couplées avec des cupules en polyéthylène (non réticulé, résine GUR 1050 et stérilisées par irradiation $\gamma$ ). D'autres couples ont également été testés pour comparaison avec la littérature.

Pour distinguer les effets du vieillissement et du frottement, les têtes Za et ZTAa ont subi uniquement du vieillissement, les têtes $\mathrm{Zw}$ et ZTAw ont subi uniquement $\mathrm{du}$ frottement sur simulateur de marche, et les Zaw et ZTAaw ont subi des alternances de frottement et vieillissement ( 1 million de cycles de frottement pour 1 heure de vieillissement). Les tests ont été menés jusqu'à 10 millions de cycles en simulateur de marche et/ou $10 \mathrm{~h}$ de vieillissement.

Le vieillissement accéléré a été mené en autoclave à $134^{\circ} \mathrm{C}$ (on estime qu'une heure de ce traitement correspond à 2-3 ans de vieillissement à $37^{\circ} \mathrm{C}$ ) [2]. Le frottement a été mené sur un simulateur de marche MTS 4 postes, en position anatomique inversée, dans du sérum bovin (dilué 4 fois dans l'eau distillée pour reproduire la

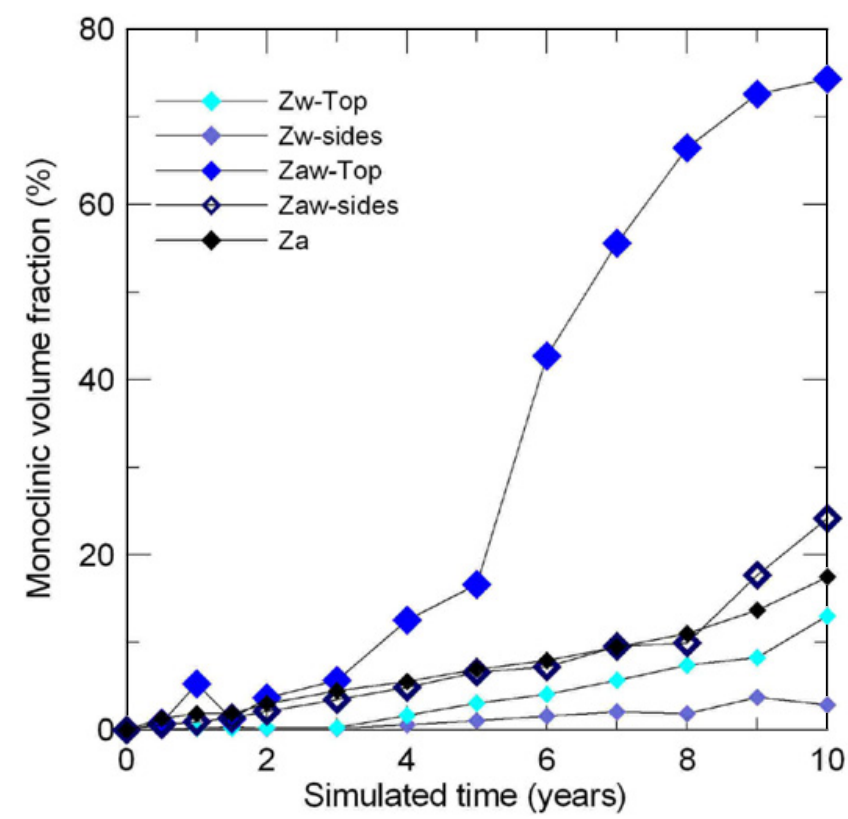

Figure 2. Evolution du taux de phase monoclinique sur les têtes en zircone.

concentration en protéines du liquide synovial humain), à une température de l'ordre de $30^{\circ} \mathrm{C}$.

Tous les millions de cycles ou toutes les heures de vieillissement, les têtes ont été soumises à une batterie de tests : mesure du taux de phase monoclinique par diffraction des rayons $X$ (en 5 points : sommet de la tête et 4 points répartis sur l'équateur), caractérisation des rugosités par interférométrie optique (5 points), mesure de perte de masse de chaque tête et de la cupule en polyéthylène associée.

Pour des raisons pratiques de comparaison entre les différentes conditions de test, on introduit la notion de «temps simulé»: 1 million de cycles de frottement ( $\mathrm{Zw}$ et ZTAw), ou 1 heure d'autoclave (Za ou ZTAa) ou 1 million de cycle plus 1 heure d'autoclave (Zaw ou ZTAaw) correspondent à un an en «temps simulé».

Le protocole est décrit plus complètement ici [3].

\section{RÉSULTATS}

\section{Perte de masse}

Les taux d'usure des différents couples sont décrits sur la figure 1. Le taux d'usure des couples de référence céramique-céramique n'est pas mesurable. Le taux d'usure des couples ZTA-PE et alumine-PE atteignent au maximum $80 \mathrm{~mm}^{3}$ par million de cycle. Le taux d'usure des couples zircone-PE est supérieur de $50 \%$ et atteint $120 \mathrm{~mm}^{3}$ par million de cycles. La perte de masse de la cupule en PE frottant contre la tête Zaw est supérieure à celle de la cupule frottant contre la tête $\mathrm{Zw}$.

\section{STABILITÉ FACE À LA TRANSFORMATION DE PHASE}

La figure 2 montre l'évolution du taux de phase monoclinique en surface des trois têtes en zircone. Elle 
montre que l'évolution est la plus rapide sur le sommet de la tête Zaw, seule zone subissant à la fois le frottement et le vieillissement (les côtés de la même tête ne frottant contre la cupule qu'occasionnellement).

Les mêmes tendances sont observées pour la rugosité des têtes. En revanche, les têtes ZTA n'évoluent pas, ni en rugosité ni en taux de phase monoclinique.

\section{DISCUSSION ET CONCLUSION}

Les résultats mettent en évidence l'accélération du vieillissement hydrothermal lorsqu'il est couplé avec le frottement. Ce phénomène pourrait être expliqué par deux causes :

(1) la notion de «frictional heating» (le frottement engendre localement une augmentation de température qui pourrait accélérer le vieillissement) doit être rejetée car les températures nécessaires à l'observation de telles vitesses de vieillissement sont bien trop élevées [3].
(2) les contraintes résiduelles jouent un rôle sur la transformation. Ce phénomène reste à quantifier.

\section{Références}

[1] National Joint Registry for England and Wales 9th annual report, 2012, ISSN 1745-1450, available on line at: http://www.njrcentre.org.uk/NjrCentre/Portals/0/Documents/England/Reports/ 9th_annual_eport/NJR 9th Annual Report 2012.pdf

[2] Chevalier J, Cales B, Drouin JM. Low temperature aging of Y-TZP ceramics. J Am Ceram Soc 1999; 82 (8) : 2150-54

[3] Gremillard L, Martin L, Chevalier J et al., Combining aging and wear to assess the durability of zirconia-based ceramic heads for total hip arthroplasty, Acta Biomaterialia 2013; (http://dx.doi.org/10.1016/j.actbio.2013. $03.030)$ 